\title{
Excitation Mechanism of Surface Plasmon Polaritons for Surface Plasmon Sensor With 1D Metal Grating Structure for High Refractive Index Medium
}

\author{
Atsushi MOTOGAITO ${ }^{1,2^{*}}$ and Yusuke ITO ${ }^{1}$ \\ ${ }^{1}$ Graduate School of Engineering, Mie University, Mie 514-8507, Japan \\ ${ }^{2}$ Iga Satellite, Mie University, Mie 518-0131, Japan \\ *Corresponding author: Atsushi MOTOGAITO E-mail: motogaito@elec.mie-u.ac.jp
}

\begin{abstract}
The excitation mechanism of surface plasmon polaritons (SPPs) in a surface plasmon sensor with a one-dimensional (1D) Au diffraction grating on a glass substrate is studied herein. The sensitivity of the sensor for application to a refractometer is also characterized. The SPPs are excited at the following two types of interface: one between the Au grating and the glass substrate and the other between the Au grating and the medium. The simulation data for the transmittance spectra and the transmittance mapping are consistent with the experimental data even when the refractive index of the solution medium is 1.700. Therefore, the excitation mechanism of the SPPs in a surface plasmon sensor is capable of detecting the medium $(n=1.700)$, in which the sensor is used and clarified.
\end{abstract}

Keywords: Surface plasmon; diffraction grating; high refractive index; chemical sensor

Citation: Atsushi MOTOGAITO and Yusuke ITO, "Excitation Mechanism of Surface Plasmon Polaritons for Surface Plasmon Sensor With 1D Metal Grating Structure for High Refractive Index Medium,” Photonic Sensors, 2019, 9(1): 11-18.

\section{Introduction}

Surface plasmon resonance (SPR) is widely used in applications, such as detection of infinitesimal materials and high-efficiency photoelectric conversion [1,2]. Compared with an enzyme-linked immunosorbent assay, SPR sensors can more easily detect the medium, in which they are used, from a small sample. SPR sensors that utilize light were first proposed by Nylander et al. in 1982 [3], and the use of a thin metal film-based sensing technique known as the attenuated total reflection method was proposed by Kretchmann [4]. Resonance results from the total reflection of light; hence, this method is affected by the relationship between the refractive index of a target medium and the contact portion of a sensor. Many harmful media such as halogen compounds (e.g., $\mathrm{Br}_{2}: n=1.640, \mathrm{SOCl}_{2}: n=1.522$ ) and heavy metal compounds (e.g., $\mathrm{C}_{14} \mathrm{H}_{16} \mathrm{~Pb}: n=$ 1.626), exhibit refractive indices greater than 1.5. The use of surface plasmon sensors for the safe detection of such media is considered advantageous because of their simple operation and ability to detect small amounts of a target for monitoring soil contamination and water pollution. For a target medium with a high refractive index $(n>1.5)$, the total reflection generally becomes impossible because of an incident angle limitation in the incidence angle of light; therefore, such a medium cannot be detected.

Received: 9 July 2018 / Revised: 31 August 2018

(C) The Author(s) 2018. This article is published with open access at Springerlink.com

DOI: $10.1007 / \mathrm{s} 13320-018-0515-8$

Article type: Regular 
A solution to this problem was demonstrated in a study using a GaP semiconductor, which had a substrate with a high refractive index $(n>1.5)$ [5]. In this case, a prism is used to allow light into the sensor and enable the use of the attenuated total reflection method for the $\mathrm{GaP}$ substrate and a thin gold film; thus, the detection of a medium with a refractive index of 1.620 is confirmed. However, even after using this structure, a medium with a refractive index greater than 1.620 is impossible to detect because of the limitation of the incidence angle. Moreover, for a device that uses a prism to allow light into the GaP substrate, the structure of the sensor must be large, which hinders device miniaturization.

We focus on a method that uses a diffraction grating as the structure of the sensor. With this method, a metal diffraction grating is used instead of a thin metal film, and the resonance is caused by the diffraction of light [6]. The diffraction grating method does not use total reflection; thus, the limitation on the incidence angle is relaxed. An additional advantage of this method is the flexibility which enables in changing the detection range and the excitation angle by modifying the period and duty ratio of the grating. Homola et al. [7, 8] investigated the sensitivity of SPR to the grating structure and the amount of processable information, confirming the high information processing capability of such structures. In addition, a target localization study shows that the differences in the region of contact between the target medium and the sensor affect its sensitivity [9].

Previous studies have primarily focused on materials with refractive indices below $1.500 \mathrm{Li}$ et al. [10] showed by calculation that a high refractive index medium $(n<2.0)$ could be detected using a metal nanograting and reported a sensitivity of $51.484^{\circ} /$ RIU. However, we experimentally demonstrated the detection of a high refractive index medium ( $n=1.546-1.700)$ using surface plasmon sensors for the first time [11]. Our previous study on a double-layer wire grid polarizer with a grating structure [12, 13] showed that extraordinary transmission [14] occurred at the interface between the metal and the contact medium. Similar phenomena may occur in the surface plasmon sensor with a 1D metal grating structure.

Based on our previous studies, these surface plasmon sensors being used as refractometers are able to measure a wide range of refractive index because of the dip angle of reflectance or the peak angle of transmittance for angular spectra, which are the angles of excitation of the surface plasmon polariton. The measurement accuracy of the film thickness using a non-contact thickness meter will be improved if the refractive index is accurately measured. Some methods can be used to measure the refractive index, such as the minimum argument method, critical angle method, and V block method for the refractometer. Compared with these measurements, the refractometer using surface plasmon sensor is superior in that prisms are unnecessary; the apparatus can be downsized, such that the measurement can be easily performed, and the measurement range is wide. Furthermore, some reports have used a refractometer using surface plasmon polariton [15-18]; however, in these methods, optical fibers have been used, and the wavelength spectra have to be measured. Most of the reports correspond to the range of refractive index less than 1.5. In contrast, in our proposed method, the dispersions of metal and the medium are not necessary because this method measures the angular spectra with one wavelength, and we can directly obtain the refractive index from the peak or dip angle for transmittance or reflectance. The range of the measured refractive index will be larger than 1.5 ; thus, the setup of the refractometer with a $1 \mathrm{D}$ grating structure is easy to use compared with using optical fiber. However, the refractometer employing the 1D metal grating structure has some issues. One is that the sensitivity at a higher refractive index is unknown, and the other is that the propagation 
mechanism of the surface plasmon polariton for the 1D metal grating structure has not been thoroughly studied.

In this study, the theoretical calculation is performed using transmittance mapping obtained by the rigorous coupled-wave analysis (RCWA) method and our experimental data.

\section{Simulation and experimental results of transmittance}

The dependence of transmittance and reflectance on the incidence angle is characterized using a red-light laser $(\lambda=635 \mathrm{~nm})$. Figure 1 shows the schematic diagram of the fabricated sensor. The thickness of $\mathrm{Au} h$ is $40 \mathrm{~nm}$. $p$ denotes the grating period. The duty ratio of the grating is 0.5 . The simulation is performed using the RCWA method with the commercial software, rcda-1d (SourceForge). In the simulation, the angle spectra of transmittance and reflectance and transmittance mapping for the incident angle and period are created.

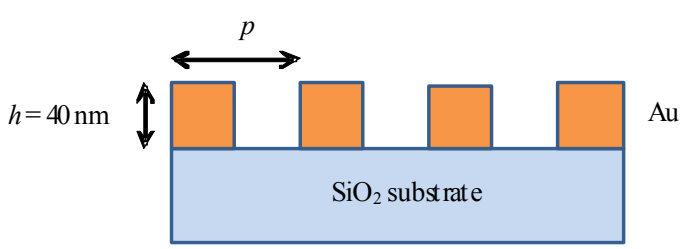

Fig. 1 Schematic diagram of the fabricated sensor structure.

We attempt to detect a mixed solution of 1-iodonaphthalene and sulfur $(n=1.700)$. Figure 2 shows the incidence angle spectra of transmittance for a medium with periods of $500 \mathrm{~nm}$ and $300 \mathrm{~nm}$. In the $500 \mathrm{~nm}$ case with sulfur, the transmittance peaks occur in the angular spectrum at $7.15^{\circ}$ and $17.35^{\circ}$. For the medium, the angle corresponding to a dip in reflectance is the same as that corresponding to a peak in transmittance. In the $300 \mathrm{~nm}$ case with sulfur, the peaks occur at $16.60^{\circ}$ and $27.35^{\circ}$. The two types of transmittance peaks in these spectra are assumed to occur because of two different modes for exciting the SPPs.

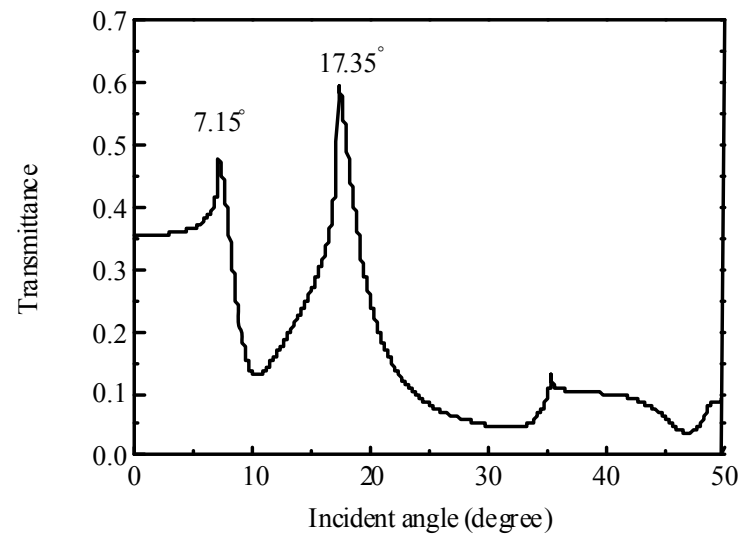

(a)

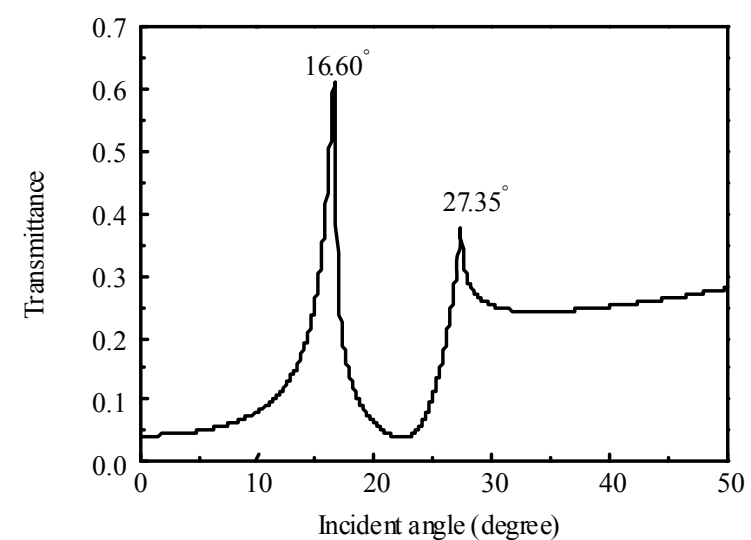

(b)

Fig. 2 Simulation results for the transmittance as a function of the incidence angle for a mixed solution of 1-iodonaphthalene and sulfur. Grid periods of (a) $500 \mathrm{~nm}$ and (b) $300 \mathrm{~nm}$.

The distribution of the magnetic field $\left|H_{y}\right|$ and the transmittance mapping, which is the relationship among the period, incidence angle, and transmittance, is created through the RCWA method to confirm the SPP origin. Figures 3(a) and 3(b) show the distribution of the magnetic field in the case of a mixed solution of 1-iodonaphthalene and sulfur for a period of $500 \mathrm{~nm}$. At $17.35^{\circ}$, the magnetic field is concentrated at the interface between $\mathrm{Au}$ grating and the solution. However, at $7.15^{\circ}$, it is concentrated on the interface between $\mathrm{Au}$ and the glass substrate. Figures 3(c) and 3(d) show the distributions of the magnetic field in the case with a period of $300 \mathrm{~nm}$. At $16.60^{\circ}$, the magnetic field is concentrated on the interface between the $\mathrm{Au}$ grating and the solution, while at $27.35^{\circ}$, it is concentrated at the interface between Au grating and 
the glass substrates. Thus, we assume that the SPPs are excited at these two types of interface.

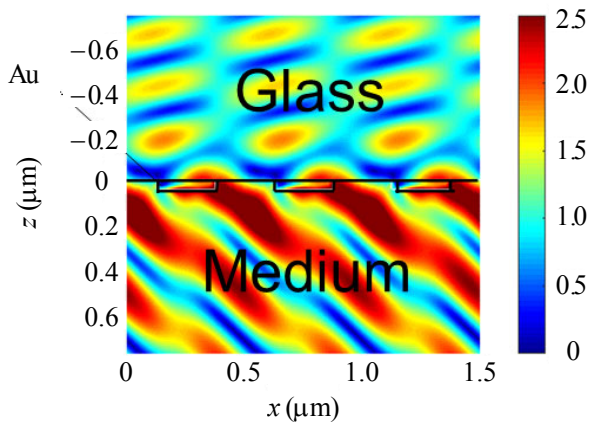

(a)

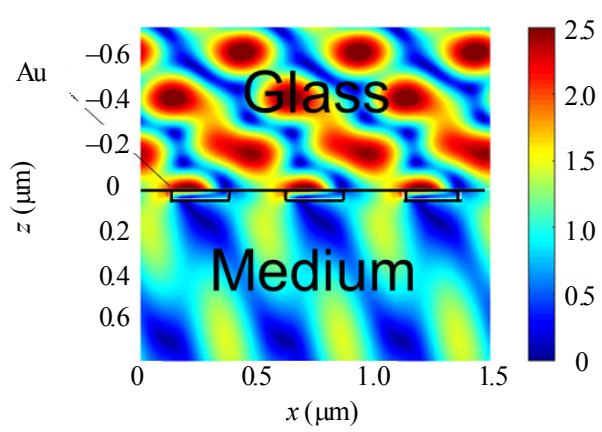

(b)

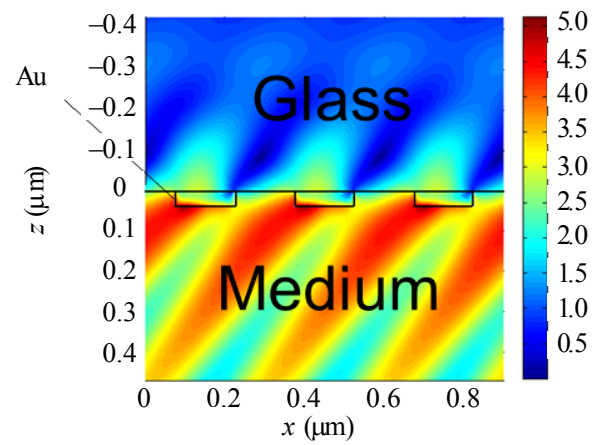

(c)

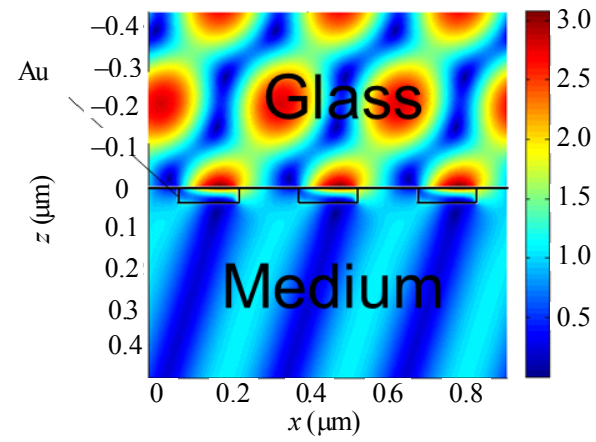

(d)

Fig. 3 Distribution of the magnetic field in the case of a mixed solution of 1-iodonaphthalene and sulfur: (a) grid period of $500 \mathrm{~nm}$ at $17.35^{\circ}$, (b) grid period of $500 \mathrm{~nm}$ at $7.15^{\circ}$, (c) grid period of $300 \mathrm{~nm}$ at $16.60^{\circ}$, and (d) grid period of $300 \mathrm{~nm}$ at $27.35^{\circ}$.
The peak angle of the transmittance is characterized for various refractive indices of the medium $(n)$. Figure 4 shows the angular spectra of the transmittance between $0^{\circ}$ and $50^{\circ}$. As shown in Figs. 2 and 3, two types of peaks appear in these spectra. One type corresponds to the interface between the $\mathrm{Au}$ grating and the glass substrate, which is called the A peak, and the other type corresponds to the interface between the Au grating and the medium, which is called the B peak. The A-peak angle is almost constant even if the refractive index of the medium is varied. However, the B-peak angle shifts when the refractive index of the medium changes. Therefore, we have to use the B peak to detect the medium using this sensor because the peak shift occurs under changes of the refractive index.

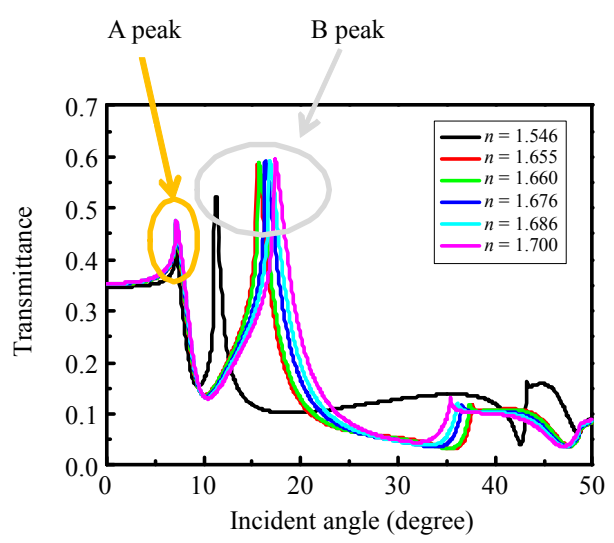

(a)

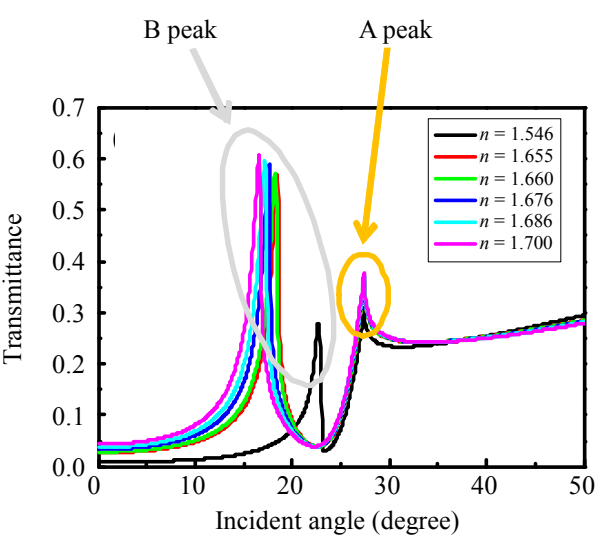

(b)

Fig. 4 Simulation results for the transmittance as a function of the incident angle spectra between $0^{\circ}$ and $50^{\circ}$ for a varying refractive index: (a) period of $500 \mathrm{~nm}$ and (b) period of $300 \mathrm{~nm}$. 
Next, the samples are fabricated using an electron beam lithography system (CABL-8000, CRESTEC). $\mathrm{Au}$ sputtering is performed after electron beam lithography. Finally, the lift off process is conducted. Figure 5 shows the scanning electron microscope (SEM) images of the fabricated sample. Following this, the dependence of transmittance and reflectance on the incident angle is characterized using a red-light laser $(\lambda=635 \mathrm{~nm})$. The transmittance is obtained by the ratio of the light power intensity in the case where no sample is present and that in the case where a sample changes the incident angle of light.

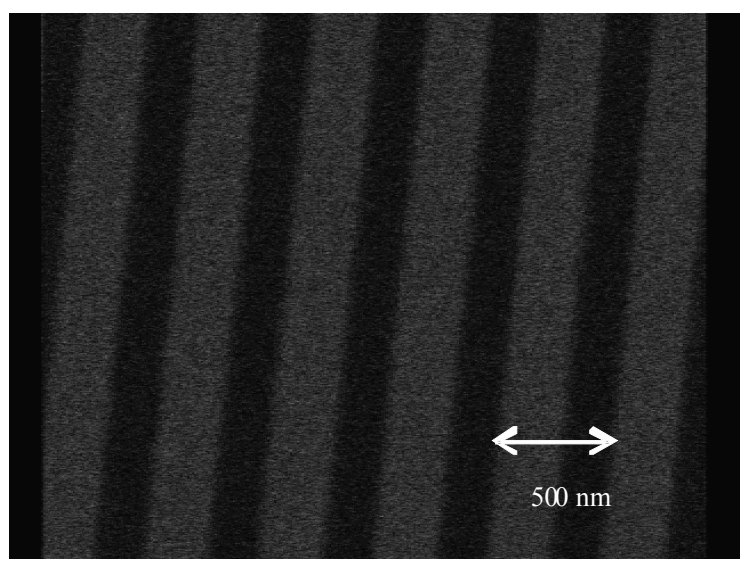

(a)

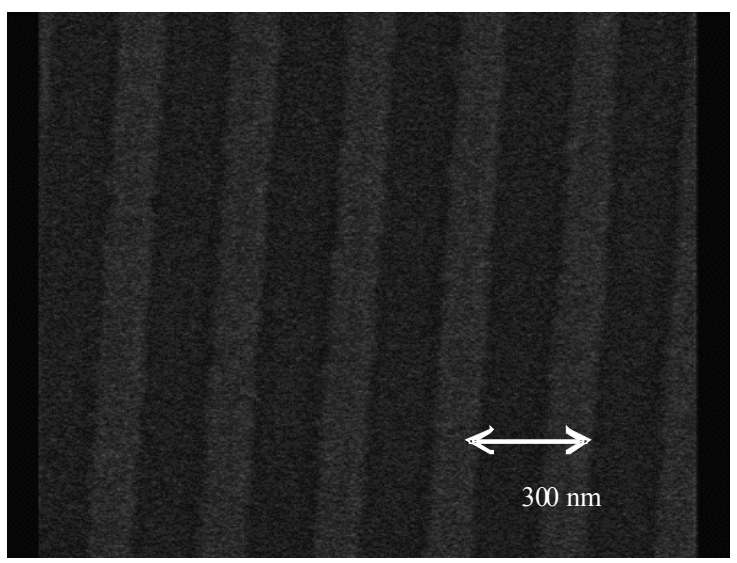

(b)

Fig. 5 SEM images of the fabricated samples: (a) period of $500 \mathrm{~nm}$ and (b) period of $300 \mathrm{~nm}$.

The incident light intensity is constant. The details of the fabrication and the optical characterization are documented in [5] and [11]. Figure 6 presents the incident angle spectra of transmittance for a medium (a mixed solution of 1-iodonaphthalene and sulfur) for periods of $500 \mathrm{~nm}$ and $300 \mathrm{~nm}$. In the $500 \mathrm{~nm}$ case with sulfur, two transmittance peaks occur in the angular spectrum at $7.56^{\circ}$ and $17.60^{\circ}$. In the $300 \mathrm{~nm}$ case, these peaks occur at $16.95^{\circ}$ and $29.19^{\circ}$. From the result of Fig. 3, these peaks are identified as the A peak and the B peak. Similar with the simulation, the full width at half maximum (FWHM) of the B peak for the $300 \mathrm{~nm}$ period is narrower than that for the $500 \mathrm{~nm}$ period; therefore, the simulation data are reasonable.

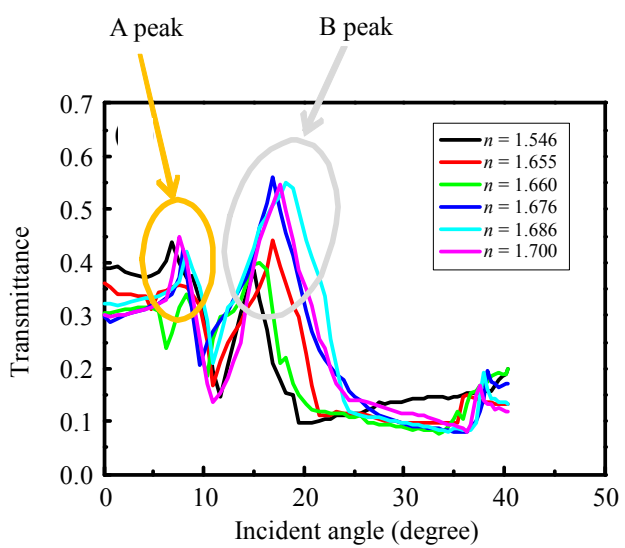

(a)

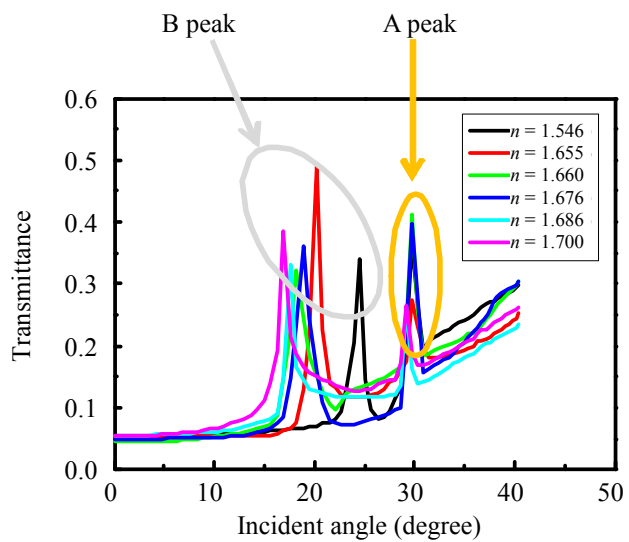

(b)

Fig. 6 Experimental results for the transmittance as a function of the incidence angle between $0^{\circ}$ and $50^{\circ}$ for a varying refractive index: (a) period of $500 \mathrm{~nm}$ and (b) period of $300 \mathrm{~nm}$.

\section{Discussion using transmittance mapping and dispersion relationship calculated via the RCWA method}

Transmittance mapping, which is the relationship of the period, incident angle, and 
transmittance, is created using the RCWA method when the refractive index of the medium is 1.70 to discuss the abovementioned results. Figure 7 shows the transmittance mapping with the relationship between the incident angle and the grating period, which includes two curves. The peak incidence angle varies with the grating period. Two kinds of curves exist in this mapping. Based on the dispersion curve of the surface plasmon for the diffractive grating, and the relationship between the surface plasmon and the incident light, they are respectively calculated as

$$
\begin{gathered}
k_{\mathrm{sp}}=(\omega / c) \sqrt{\varepsilon_{1} \varepsilon_{2} / \varepsilon_{1}+\varepsilon_{2}}+m K \\
k \sin \theta=\left(2 \pi n / \lambda_{0}\right) \sin \theta=k_{\mathrm{sp}}
\end{gathered}
$$

where $\varepsilon_{1}$ and $\varepsilon_{2}$ are the permittivities for metal and insulator, respectively, $\omega$ is the angular frequency of light, $K$ is the wave number of the grating, $m$ is the arbitrary integer, $c$ is the velocity of light, $k$ is the wave number of the incident light, $n$ is the refractive index of the medium, $\lambda_{0}$ is the wavelength in vacuum, and $\theta$ is the incident angle of light.

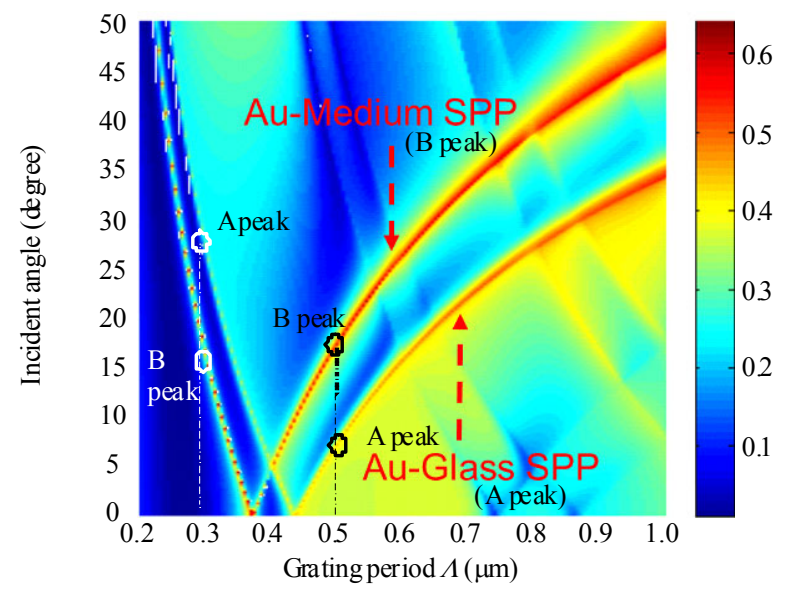

Fig. 7 Transmittance mapping obtained using the RCWA method.

One curve is related to the SPP excitation at the interface between $\mathrm{Au}$ and glass (corresponding to the A peak), while the other is related to that at the interface between the $\mathrm{Au}$ grating and the medium (corresponding to the B peak) [13]. The former curve related to the A peak is located almost at the same position because the peak angle does not change with the refractive index. However, the curve related to the $B$ peak changes with the refractive index. The experimental data are plotted in the mapping and consistent with these curves. In Figs. 4 and 6, the reason for the A peak on the left for $500 \mathrm{~nm}$ and that for the B peak on the left for the $300 \mathrm{~nm}$ period gratings are the angle of the A peak for $500 \mathrm{~nm}$ being lower than that for the B peak and the angle of the A peak for $300 \mathrm{~nm}$ being higher than that for the B peak (Fig. 7). Similar results are obtained using the transmittance mapping in the wire grid polarizer [19]. Therefore, in the SPP sensor with an $\mathrm{Au}$ grating structure, the SPPs are excited at the two kinds of interface.

We calculate the dispersion curves, $k_{\mathrm{sp}}$ and $k \sin \theta$, of a propagation-type surface plasmon based on the simulation using the RCWA method.

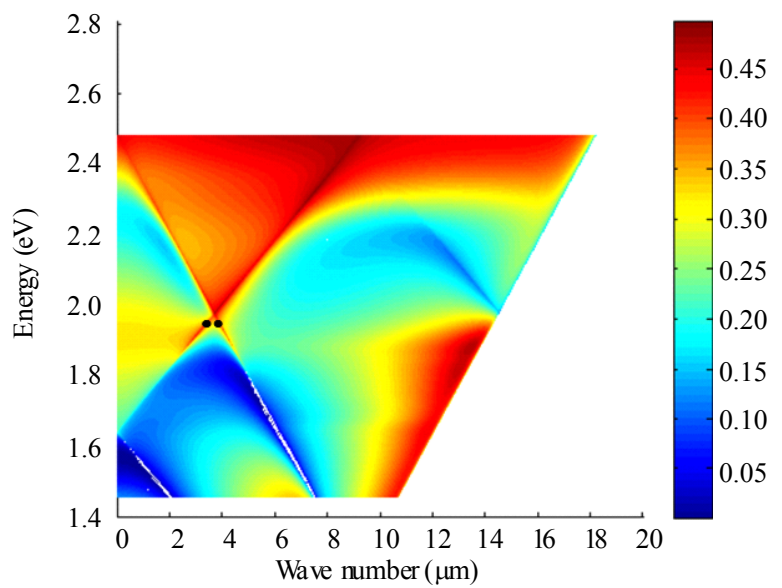

(a)

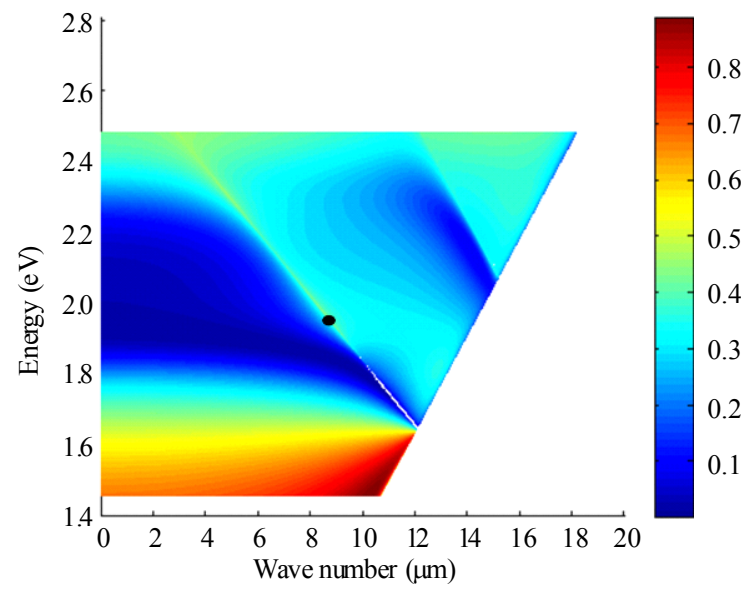

(b)

Fig. 8 Transmittance mapping with the dispersion curve obtained using the RCWA method: (a) period of $500 \mathrm{~nm}$ and (b) period of $300 \mathrm{~nm}$. 
Figure 8 shows the transmittance mapping with the relationship between the light energy and the wavenumber of the SPP. The ordinate axis indicates the energy level, while the abscissa axis indicates the wave number. The line that slantingly develops from $\sim 11(/ \mu \mathrm{m})$ is a light line. Figure 8 presents the white locuses. The dispersion curves are almost identified using (1), and the SPP-excitation point (denoted by a dot) can be obtained from the cross-section between the dispersion curve and the straight line $(h v=1.95 \mathrm{eV})$. The SPP-excitation angle can be obtained using (2). This angle is mostly consistent with the experimental and simulation data; therefore, the propagation-type SPP is excited in this structure.

\section{Conclusions}

In this study, a theoretical calculation is performed using transmittance mapping obtained via the RCWA method and our experimental data to consider the application of a surface plasmon sensor to a refractometer.

The transmittance mapping shows that the SPPs are excited to two types of interface, namely the Au-glass substrate and the Au-medium. The latter should be used to detect the medium using the SPPs. We confirm the detection of the medium of the refractive index as $n=1.700$. The mechanism of this sensor is also cleared using transmittance mapping. Therefore, the potential for the application of a refractometer in the high-refractive index region is obtained.

\section{Acknowledgment}

This work is supported by Grants in Aid for Scientific Research of Japan Society for the Promotion of Science (JSPS, KAKENHI, Grant Nos. 26390082 and 15H03556).

We would like to thank Prof. Kazumasa Hiramatsu and Prof. Hideto Miyake in Mie University for useful discussions. We would also thank Editage (www.editage.jp) for English language editing.
Open Access This article is distributed under the terms of the Creative Commons Attribution 4.0 International License (http://creativecommons.org/licenses/by/4.0/), which permits unrestricted use, distribution, and reproduction in any medium, provided you give appropriate credit to the original author(s) and the source, provide a link to the Creative Commons license, and indicate if changes were made.

\section{References}

[1] K. Hering, D. Cialla, K. Ackermann, T. Dörfer, R. Möller, H. Schneidewind, et al., "SERS: a versatile tool in chemical," Analytical and Bioanalytical Chemistry, 2008, 390(1): 113-124.

[2] H. A. Atwater and A. Polman, "Plasmonics for improved photovoltaic devices," Nature Materials, 2010, 9: 205-213.

[3] C. Nylander, B. Liedberg, and T. Lind, "Gas detection by means of surface plasmon resonance," Sensors and Actuators, 1982-1983, 3: 79-88.

[4] E. Kretschmann, "Die bestimmung optischer konstanten von metallen durch anregung von oberflächenplasmaschwingungen," Zeitschrift für Physik A Hadrons and Nuclei, 1971, 241(4): 313-324.

[5] A. Motogaito, S. Nakamura, J. Miyazaki, H. Miyake, and K. Hiramatsu, "Using surface-plasmon polariton at the $\mathrm{GaP}-\mathrm{Au}$ interface in order to detect chemical species in high-refractive-index media," Optics Communications, 2015, 341: 64-68.

[6] H. Kano and S. Kawata, "Grating-coupled surface plasmon for measuring the refractive index of a liquid sample," Japanese Journal of Applied Physics, 1995, 34(1): 331-335.

[7] J. Homola, I. Koudela, and S. S. Yee, "Surface plasmon resonance sensors based on diffraction gratings and prism couplers: sensitivity comparison," Sensors and Actuators B, 1999, 54(1-2): 16-24.

[8] J. Dostálek, J. Homola, and M. Miler, "Rich information format surface plasmon resonance biosensor based on array of diffraction gratings," Sensors and Actuators B, 2005, 107(1): 154-161.

[9] M. Chamtouri, M. Sarkar, J. Moreau, M. Besbes, H. Ghalila, and M. Canva, "Field enhancement and target localization impact on the biosensitivity of nanostructured plasmonic sensors," Journal of the Optical Society of America B, 2014, 31(5): 1223-1231.

[10] X. F. Li, W. Peng, Y. L. Zhao, Q. Wang, and J. L. Wei, "A subwavelength metal-grating assisted sensor of Kretschmann style for investigating the sample with high refractive index," Chinese Physics B, 2016, 25(3): 037303-1-037303-4.

[11] A. Motogaito, S. Mito, H. Miyake, and K. Hiramatsu, "Detecting high-refractive-index media using surface plasmon sensor with one-dimensional metal 
diffraction grating," Optics and Photonics Journal, 2016, 6(7): 164-170.

[12] A. Motogaito, Y. Morishita, H. Miyake, and K. Hiramatsu, "Extraordinary optical transmission exhibited by surface plasmon polaritons in a double-layer wire grid polarizer," Plasmonics, 2015, 10(6): 1657-1662.

[13] A. Motogaito, T. Nakajima, H. Miyake, and K. Hiramatsu, "Excitation mechanism of surface plasmon polaritons in a double-layer wire grid structure," Applied Physics A, 2017, 123(12): 729-1-729-5.

[14] T. W. Ebbesen, H. J. Lezec, H. F. Ghaemi, T. Thio, and P. A. Wolff, "Extraordinary optical transmission through sub-wavelength hole arrays," Nature, 1998, 391: 667-669.

[15] Y. A. Qazwini, A. S. M. Noor, Z. A. Qazwini, M. H. Yaacob, S. W. Harun, and M. A. Mahdi, "Refractive index sensor based on SPR in symmetrically etched plastic optical fibers," Sensors and Actuators A,
2016, 246: 163-169.

[16] J. Zhao, S. Q. Cao, C. R. Liao, Y. Wang, G. J. Wang, $\mathrm{X}$. Z. Xu, et al., "Surface plasmon resonance refractive sensor based on silver-coated side-polished fiber," Sensors and Actuators B, 2016, 230: 206-211.

[17] L. T. Ji, X. Q. Sun, G. B. He, Y. Liu, X. B. Wang, Y. J. $\mathrm{Yi}$, et al., "Surface plasmon resonance refractive index sensor based on ultraviolet bleached polymer waveguide," Sensors and Actuators B, 2017, 244: 373-379.

[18] H. Apriyanto, G. Ravet, O. D. Bernal, M. Cattoen, H. C. Seat, V. Chavagnac, et al., "Comprehensive modeling of multimode fiber sensors for refractive index measurement and experimental validation," Scientific Report, 2018, 8: 5912-1-5912-13.

[19] Z. C. Ye, J, Zheng, S, Sun, S. J. Chen, and D. H. Liu, "Compact color filter and polarizer of bilayer metallic nanowire grating based on surface plasmon resonances," Plasmonics, 2013, 8(2): 555-559. 